\title{
REARRANGEMENTS OF CONDITIONALLY CONVERGENT REAL SERIES WITH PREASSIGNED CYCLE TYPE
}

\author{
JOHN HOWARD SMITH
}

\begin{abstract}
For any conditionally convergent real series, any real number $r$, and any infinite cycle type, there is a permutation of the indices, of the given cycle type, which makes the series converge to $r$.
\end{abstract}

One result usually mentioned and sometimes proved when conditionally convergent series are first discussed is Riemann's theorem that for any real number $r$ there is a permutation of the indices so that the renumbered series converges to $r$. The usual method of renumbering gives no control over the type of the permutation (in the sense of disjoint cycle decomposition). John Kelly has asked whether we may, for example, take the permutations to be of order 2. In this note we investigate whether the job can be done with a permutation with prescribed cycle type, and show that, subject to the obvious condition that the cycle type allow for moving infinitely many indices, it can be.

Let $N$ be the natural numbers $\{1,2, \ldots\}$; let $N_{0}=N \cup\{0\}, N_{\infty}=N \cup$ $\{\infty\}, N_{0 \infty}=N \cup\{0, \infty\}$. A cycle type is a function $t$ from $N_{\infty}$ to $N_{0 \infty}$; it is to be thought of as specifying how many 1-cycles (fixed points), 2-cycles, etc., including infinite cycles, a permutation of a (countable) set is to have in its disjoint cycle decomposition. If $t(y)=x$, we shall say that $t$ has $x$ $y$-cycles.

Clearly a permutation $\sigma$ moves only finitely many elements if and only if its cycle type, $t_{\sigma}$, satisfies :

(1) $t_{\sigma}(\infty)=0$;

(2) $t_{\sigma}(n)<\infty$ for any $n \in N, n \neq 1$;

(3) $t_{\sigma}(n)=0$ for all but finitely many $n$.

Such a cycle type will be called finite.

Theorem. If $\Sigma a_{n}$ is a conditionally convergent series of real numbers,

Received by the editors October 2, 1973.

AMS (MOS) subject classifications (1970). Primary 40 A05; Secondary 05A99.

Key words and phrases. Conditionally convergent series, cycle type of permutation. 
$c$ a real number, and $t$ an infinite cycle type, then there is a permutation of $N$, with $t_{\sigma}=t$, such that $\Sigma_{N} a_{\sigma(n)}=c$.

Before proving the Theorem we establish some special cases.

Lemma.1. Suppose that $\Sigma_{N} a_{n}$ converges conditionally, and $\Sigma_{I} a_{n}, I \subset$ $N$, is a divergent subseries of nonnegative terms. Suppose further that $t$ is an infinite cycle type with $t(1)=\infty, t(\infty)=0$. Then for any nonnegative real number $d$, there is a permutation $\sigma$ on $N$, of type $t$, fixing all elements of $N-I$, such that $\Sigma_{N} a_{\sigma(n)}=\left(\Sigma_{N} a_{n}\right)-d$.

Proof. We may assume $N-I$ is infinite; if not take $I^{\prime} \subset I, N-I^{\prime}$ infinite with $I^{\prime}$ satisfying the same properties.

Now rearrange the series $1-1+1 / 2-1 / 2+1 / 3-1 / 3 \cdots$ so that it converges to $d$. Do this in such a way that

(1) $1 / m$ occurs before $-1 / m$ for all $m \in N$.

(2) If $m>m^{\prime}$ then $1 / m^{\prime}$ occurs before $1 / m$ and $-1 / m^{\prime}$ before $-1 / m$. Call the rearranged series $\Sigma_{N} r_{n}$.

We now construct certain finite subsets, $I( \pm m), m \in N$, of $I$ which occur in the same "order" as the corresponding $\pm 1 / m$ in $\Sigma r_{n}$. The sum of $a_{n}$ over $I(m)$ will be approximately $1 / m$, that over $l(-m)$ approximately zero. We then construct a permutation with the given cycle type which interchanges the elements of $l(m)$ with some of those of $l(-m)$. The effect will be to decrease the sum over $l(m)$ by about $1 / m$ and increase the (later) terms corresponding to $I(-m)$ by the same amount. Because of the order of the sets $I( \pm m)$, the net effect on the series turns out to be a decrease of $d$ in the total sum.

The cycle type $t$ tells us that the desired permutation will consist of infinitely many 1-cycles and infinitely many nontrivial finite cycles. Let the length $s$ of the latter be $s_{1}, s_{2}, \cdots$. Thus each integer $n \neq 1$ occurs $t(n)$ times in this sequence.

Now we construct $l( \pm m) \subset I$ so that:

(1) Any two of the $I( \pm m)$ are disjoint.

(2) $\left|\left(\Sigma_{I(m)} a_{k}\right)-1 / m\right|<2^{-m}$.

(3) $\left|\Sigma_{I(-m)} a_{n}\right|<2^{-m}$.

(4) If $1 / x$ comes before $1 / y$ in $\Sigma r_{n}$, then every element of $I(x)$ is less than every element of $l(y)$. (Note that this implies (1).)

(5) For every $m_{1} \in N, \Sigma_{1}^{m} 1|I(-m)|=\Sigma_{1}^{m} 2\left(s_{m}-1\right)$, where $m_{2}=\Sigma_{1}^{m} 1|I(m)|$.

The blocks are constructed following the order of $\Sigma r_{n}$ as indicated in (4). There will be no difficulty satisfying (1), (2) and (3) since the terms 
$a_{n}, n \in I$, approach zero but their sum diverges. The effect of (5) is to specify $|I(-m)|$ in terms of $\left|I\left( \pm m^{\prime}\right)\right|, m^{\prime}<m$, which, by the construction of $\Sigma r_{n}$ will occur before $I(-m)$, so the blocks may in fact be determined recursively.

The purpose of condition (5) is so that if the elements of $U l_{m}$ are numbered consecutively, and if the $k$ th occurs in $l(m)$, there will be $s_{k}-1$ elements in $I(-m)$ with which we can combine it to make an $s_{k}$-cycle. We construct $\sigma$ to do this for all $k$, and to leave all other elements of $N$ fixed. Thus $\sigma$ has the desired cycle type.

Now we show that $\Sigma_{N} a_{n}-\Sigma_{N} a_{\sigma(n)}=d$ by comparing $\Sigma_{K} a_{\sigma(n)}$ with $\Sigma_{K} a_{n}, K=\{1,2, \cdots, k\}$.

For those $m$ for which $(I(m) \cup I(-m)) \subset K$, the sums over $(I(m) \cup I(-m)) \cap$ $K$ are the same. For those $m$ for which $I(m) \subset K, I(-m) \subset N-K$, the first sum over $I(m) \cap K$ will be less than the second sum by an amount within $2 \cdot 2^{-m}$ of $1 / m$, hence the sums over all such sets will differ by a partial sum of the series $\Sigma r_{n}$ plus something which will be small if $k$ is large. Finally for the (at most one) set $l( \pm m$ ) which meets both $K$ and $N-K$, both sums taken over $l( \pm m) \cap K$ will be small if $k$ is large. Since this accounts for all indices at which the two sums may differ, we see that as $k \rightarrow \infty$, their difference approaches $\Sigma r_{n}=d$.

Lemma 2 is a variation of Lemma 1 where the conditions on $t$ are replaced by $t(1)=\infty ; t(n)=0, n \neq 1, t(\infty)=1$.

The spirit of the construction is the same except that we take $|I(-m)|=$ $|I(m)|$. Then $\sigma$ takes the $k$ th element of $I(m)$ to the $k$ th of $I(-m)$, and the $k$ th of $I(-m)$ to the $(k+1)$ st of $I(m)$, except that it takes the last of $I(-m)$ to the first of $I(m+2)$ if $m$ is odd, the first of $I(m-2)$ if $m$ is even and $m \neq 2$, the first of $I(1)$ if $m=2$. The verification that this does the job is left to the reader.

Lemma 3. For any infinite $l \subset N$ and any infinite cycle type there is a permutation $\sigma$ of type $t$ on $I$ such that if $\sigma^{\prime}$ is the extension of $\sigma$ to $N$ defined by $\sigma^{\prime}(n)=n$ for $n \in N-l, \Sigma_{N} a_{n}=\Sigma_{N} a_{\sigma^{\prime}(n)}$.

Proof. Take an absolutely convergent subseries $\Sigma_{I} a_{n}$ and rearrange in any manner to use up cycles so there remains to be used an infinite type with at most one infinite cycle. This may then be made to act "harmlessly" on the remaining elements using the main result of [1].

Finally we prove the main theorem. If $t$ is the sum of two infinite cycle types, things are easy; we use some of the cycle type to achieve the de- 
sired change using Lemmas 1 or 2 (or their analogues if an increase is desired). We then use up the rest in some "harmless" manner by Lemma 3, taking care not to leave too many fixed points if $t(1) \neq \infty$.

In the remaining case, $t$ has 1 infinite cycle and finitely many nontrivial finite cycles. We may use up the latter at the beginning, and, if $t(1)=$ $\infty$, apply Lemma 2 directly. If $t(1)<\infty$, then the construction of Lemma 2 must be slightly refined to avoid an excess of fixed points. The details are not difficult.

The partial extension of Riemann's theorem to conditionally convergent series of complex numbers, and more generally of vectors in $n$-space, has been extensively studied by Steinitz [4]. It might be interesting to investigate which, if any, of his results are affected by restrictions on the cycly type of the permutations involved. It might also be interesting to see whether there are any restrictions on cycle type in other "rearranging" theorems in analysis, such as von Neumann's theorem that if two sequences in a compact metric space have the same set of cluster points, then there is a rearrangement of one (permutation of the indices) such that the distance between corresponding terms approaches zero.

\section{REFERENCES}

1. R. P. Agnew, Permutations preserving convergence of series, Proc. Amer. Math. Soc. 6 (1955), 563-564. MR 17, 146.

2. P. R. Halmos, Permutations of sequences and the Schröder-Bernstein theorem, Proc. Amer. Math. Soc. 19 (1968), '509-510. MR 37 \#2179.

3. J. von Neumann, Characterisierung des Spektrums eines Integraloperators, Actualités Sci. Indust., no. 229, Hermann, Paris, 1935, pp. 11-12.

4. E. Steinitz, Bedingt konvergente Reihen und konvexe systeme, J. Reine Angew. Math. 143 (1913), 128-175; ibid. 144 (1914), 1-40; 146 (1916), 1-52.

DEPARTMENT OF MATHEMATICS, BOSTON COLLEGE, CHESTNUT HILL, MASSACHUSETTS 02167 